\title{
Anharmonic Damping of Terahertz Acoustic Waves in a Network Glass and Its Effect on the Density of Vibrational States
}

\author{
G. Baldi, ${ }^{1, *}$ V. M. Giordano, ${ }^{2,3}$ B. Ruta, ${ }^{4}$ R. Dal Maschio, ${ }^{5}$ A. Fontana, ${ }^{6,7}$ and G. Monaco ${ }^{6}$ \\ ${ }^{1}$ IMEM-CNR Institute, Parma Science Park, I-43124 Parma, Italy \\ ${ }^{2}$ Institut Lumière Matière, UMR 5306 Université Lyon 1-CNRS, Université de Lyon, F-69622 Villeurbanne, France \\ ${ }^{3}$ SIMAP, UJF, CNRS, INP Grenoble, F-38402 St. Martin d'Heres, France \\ ${ }^{4}$ European Synchrotron Radiation Facility, BP220, F-38043 Grenoble, France \\ ${ }^{5}$ Industrial Engineering Department, Trento University, I-38123 Trento, Italy \\ ${ }^{6}$ Physics Department, Trento University, I-38123 Povo, Trento, Italy \\ ${ }^{7}$ IPCF-CNR, UOS of Roma, clo Roma University “La Sapienza," I-00185 Roma, Italy
}

(Received 6 December 2013; published 25 March 2014)

\begin{abstract}
We report the observation, by means of high-resolution inelastic x-ray scattering, of an unusually large temperature dependence of the sound attenuation of a network glass at terahertz frequency, an unprecedentedly observed phenomenon. The anharmonicity can be ascribed to the interaction between the propagating acoustic wave and the bath of thermal vibrations. At low temperatures the sound attenuation follows a Rayleigh-Gans scattering law. As the temperature is increased the anharmonic process sets in, resulting in an almost quadratic frequency dependence of the damping in the entire frequency range. We show that the temperature variation of the sound damping accounts quantitatively for the temperature dependence of the density of vibrational states.
\end{abstract}

DOI: 10.1103/PhysRevLett.112.125502

When the wavelength of a sound wave is sufficiently large with respect to the average size of the elastic modulus fluctuations, an amorphous solid behaves in a way similar to a continuous elastic medium. Since the elasticity of a glass is typically correlated on the nanometer scale [1-3], the wave propagation is influenced by the heterogeneous elasticity of the material when the frequency reaches the terahertz range [3]. A proper understanding of the propagation of elastic waves in random media is of great interest for many diverse fields. Examples are the attenuation of seismic waves in Earth's lithosphere [4,5] or the ability to tune the thermal conductivity of a thermoelectric material $[6,7]$. From a more fundamental perspective, the main unsolved problem concerns the nature of the vibrational modes of glasses in the few terahertz frequency range and the associated thermal transport properties [8-10]. The reduced density of vibrational states (RDOS) $g(\omega) / \omega^{2}$ deviates from the Debye prediction and presents a peak, usually termed the boson peak (BP). This excess of states is often related to the known low temperature anomalies of the specific heat and of the thermal conductivity [8].

Various models have been proposed to explain the BP anomaly, models that we can classify in three main groups, without the claim of being exhaustive. A first group includes those models, such as the soft potential model $[11,12]$ and the jamming scenario [13], that associate the BP to quasilocalized soft modes. A second class of models relates the excess of states to the elastic heterogeneities of the glass. For instance, Ref. [14] suggests the appearance of localized modes close to the elastic heterogeneities, while
PACS numbers: 63.50.Lm, 62.30.+d, 62.65.+k, 66.70.Hk

the model of Refs. [15,16], recently extended to also take into account the role of anharmonic interactions [17], demonstrates that a distribution of random elastic constants can give rise to a peak in the RDOS. As a third class we mention those models where the BP is explained as a broadened first van Hove singularity, shifted to lower frequency by the disorder [18] and by the difference in mass density between the glass and the corresponding crystalline structure $[19,20]$.

The dynamic structure factor $S(Q, \omega)$ provides richer information on the glass vibrations with respect to the density of states (DOS). From its knowledge one can obtain the dispersion and damping of the pseudoacoustic excitations. Many inelastic x-ray scattering (IXS) experiments [21-26] have shown that the sound attenuation $\Gamma$ of glasses at frequencies $\nu$ close to but smaller than that of the $\mathrm{BP}$ is dominated by an "elastic" scattering process, which grows as the fourth power of the frequency. The origin of the strong scattering regime, $\Gamma \sim \nu^{4}$, is debated in the literature [9,27-32], but recent experiments [3] and theories [33] support the interpretation, dating back to Rayleigh [34], of sound wave scattering from elastic modulus fluctuations. All of the IXS experiments performed up to now have, to the best of our knowledge, supported the idea that the sound damping at these frequencies is not affected by anharmonic processes [35].

Besides the scattering by random inhomogeneities, other mechanisms, related to anharmonic or relaxational processes, can attenuate a sound wave. It is well known that quantum tunneling or thermally activated relaxation 
processes between two level states give rise to sound attenuation in glasses at relatively low temperatures and frequencies [36]. Purely anharmonic processes are usually present at higher temperatures and frequencies [37] and are qualitatively similar to the main attenuation processes in crystalline solids [38,39]. In crystals the attenuation at sufficiently low frequencies can be computed in the Akhieser approximation [40], where the sound wave is considered to interact with the assembly of thermal vibrations as a whole. Very little is known about the anharmonicity of vibrations in glasses in the terahertz frequency regime. Experiments on ethanol indicate that glass has higher Grüneisen constants than the corresponding crystalline phases [41]. A model for the third and fourth order anharmonicity in glasses suggests that these processes are responsible for the quasielastic scattering in the glass transition region and above [42]. Some evidences of a temperature dependence of the intermediate scattering function around the BP region have also been reported $[43,44]$, based on molecular dynamics simulations of vitreous silica at high temperatures $\left(T>T_{g}\right)$. However, other molecular dynamics simulations studies of the same system [45] support the hypothesis that the BP vibrations in the glass have a predominantly harmonic character.

In this Letter we report the first experimental evidence of a purely anharmonic process responsible for a marked temperature variation of the sound attenuation of a network glass at terahertz frequency. We then study in detail the relation between the sound propagation and the density of states, showing that the temperature variation of the sound damping can account quantitatively for the temperature dependence of the DOS.

The studied glass is a sodium silicate, $60 \% \mathrm{SiO}_{2}-$ $40 \% \mathrm{Na}_{2} \mathrm{O}$, that has been chosen for its relatively low glass transition temperature $\left(T_{g} \sim 694 \mathrm{~K}\right.$ [46]) and because of its high $\mathrm{x}$-ray scattering cross section, which allowed us to obtain high-quality IXS spectra. Details of the sample preparation can be found in Ref. [47]. The IXS experiment has been performed at the ID16 beam line of the ESRF in Grenoble (France). The spectrometer has been operated at the $(12,12,12)$ reflection of the main Si crystal monochromator $\left(E_{\mathrm{in}} \sim 23.7 \mathrm{keV}\right)$, leading to an energy resolution $\Delta E \sim 1.5 \mathrm{meV}$, corresponding to a frequency resolution $\Delta \nu \sim 0.36 \mathrm{THz}$.

The IXS spectra are collected at fixed momentum transfer $Q$ values as a function of frequency. The IXS cross section is proportional to the dynamic structure factor $S(Q, \omega)$ convoluted with the instrumental resolution function $R(\omega)$. The proportionality factor, which is a function of the analyzer reflectivity, the detectors efficiency, and the atomic form factors, is determined using the second moment sum rule [48]. At sufficiently low $Q$ 's the $S(Q, \omega)$ of a glass can be modeled as a delta function for the elastic line and a damped harmonic oscillator (DHO) for the inelastic component. Further details of the data analysis can be found in the
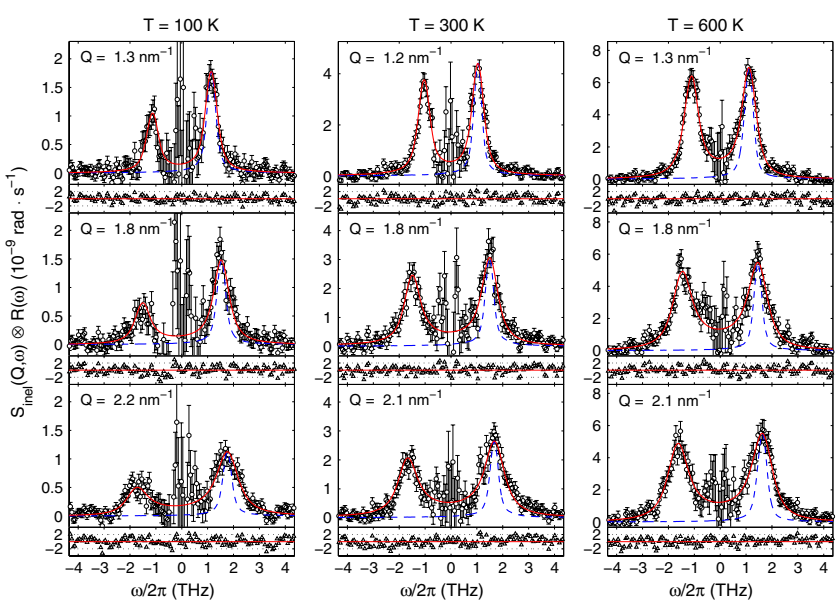

FIG. 1 (color online). Inelastic part of the IXS spectra at selected $Q$ values. The three columns correspond to the indicated temperatures. The continuous red line is the best fitting DHO function. The dashed blue line is the resolution function plotted at the position of the Stokes peak. The residues are plotted in standard deviation units below each spectrum.

Supplemental Material [49]. Figure 1 shows the inelastic part of the measured intensity for a selection of $Q$ 's at three temperatures below $T_{g}$. The excellent agreement between the model and the data can be inferred from the low values of the residues, shown below each spectrum.

At variance with the common belief that the sound attenuation in glasses at terahertz frequency is temperature independent [35], here we find evidence that $\Gamma$ is markedly affected by temperature. This observation, reported here for the first time, is discernible directly from Fig. 1. At the lowest temperature and $Q$ (the panel in the upper left corner of the figure), the width of the spectrum is close to the instrumental resolution (dashed line) and $\Gamma$ approaches the lowest measurable value. As the temperature is increased, the spectrum broadens with respect to the resolution function, implying a temperature dependence of $\Gamma$. The relative variation of $\Gamma$ with temperature gets smaller as $Q$ is increased, as shown by the second and third rows of Fig. 1 .

The inelastic peak position $\Omega(Q)$ and its full width at half maximum $\Gamma(Q)$, obtained by a minimization of the $\chi^{2}$ between the DHO model and the spectra, are shown in Fig. 2 as open symbols. It is worth noting here that $\Omega$ is the peak of the longitudinal current:

$$
J_{L}(Q, \omega)=\frac{\omega^{2}}{Q^{2}} S(Q, \omega),
$$

while the peak of $S(Q, \omega)$ is found, for the DHO model, at $\bar{\omega}=\sqrt{\Omega^{2}-\Gamma^{2} / 2}[50]$. By comparing the dynamics of a glass and the corresponding polycrystal, we have recently shown [3] that the longitudinal pseudoacoustic branch is located close to $\bar{\omega}$, while $\Omega$ is shifted to higher frequencies when opticlike modes set in. Figure 2(a) shows the different $Q$ dependence of $\Omega(Q)$ and $\bar{\omega}(Q)$, open and closed 

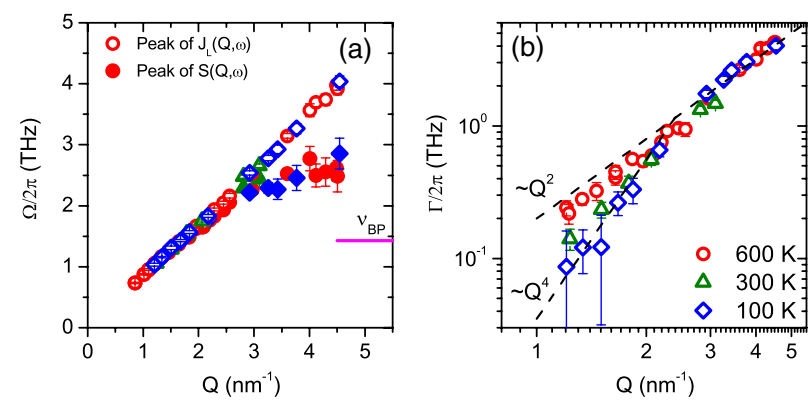

FIG. 2 (color online). (a) Dispersion of the quasilongitudinal branch. The open symbols refer to the parameter $\Omega$ of the DHO, the peak of the longitudinal current. The full symbols are the peak positions of the $S(Q, \omega), \bar{\omega}$. Red circles, $600 \mathrm{~K}$; green triangles, $300 \mathrm{~K}$; blue diamonds, $100 \mathrm{~K}$. The pink horizontal line indicates the BP frequency $\nu_{\mathrm{BP}}$. (b) $Q$ dependence of the sound attenuation. The dashed lines are guides for the eyes at the indicated slopes. Symbols as in (a).

symbols, respectively. While $\Omega(Q)$ is approximately linear in the entire explored $Q$ range, $\bar{\omega}(Q)$ deviates from linearity when $Q \geq 3 \mathrm{~nm}^{-1}$ and reaches a maximum value $\bar{\omega}_{\max } \sim 2.7 \mathrm{THz}$. It is reasonable to assume that also in our case the separation between $\Omega$ and $\bar{\omega}$ is related to the emergence of opticlike modes, which give rise to a broadening of the spectrum. If $\bar{\omega}$ is assumed to be close to the frequency of the quasilongitudinal acoustic branch, the dispersion of the quasitransverse branch can be estimated from the values of the macroscopic longitudinal and transverse sound velocities, $v_{L}$ and $v_{T}$. In particular, the maximum of the quasitransverse branch is expected to be located at $\omega_{\max }^{T} \sim\left(v_{T} / v_{L}\right) \bar{\omega}_{\max } \sim 1.5 \mathrm{THz}$, very close to the BP position $\nu_{\mathrm{BP}}$ [47]. Our analysis is thus consistent with other evidences [18-20] suggesting that the BP is a smeared first van Hove singularity of the TA phonons.

The variation with temperature of the $Q$ dependence of $\Gamma$ is shown in Fig. 2(b). The sound attenuation at low temperature experiences a fast increase with $Q$ at low $Q$ 's, in agreement with the Rayleigh scattering law, $\Gamma \sim Q^{4}$. On the contrary, the $600 \mathrm{~K}$ data follow a $\sim Q^{2}$ law in the entire explored $Q$ range but with a slightly higher slope at high $Q$ 's.

The temperature and frequency dependence of the sound attenuation can be modeled as the sum of an anharmonic term and a term accounting for the Rayleigh-Gans [51] scattering from fluctuations in the local elastic modulus:

$$
\Gamma=\Gamma_{\mathrm{AK}}+\Gamma_{\mathrm{RG}}=\frac{\gamma^{2} c_{V} v_{L} T}{2 v_{D}^{3}} \frac{\omega^{2} \tau}{1+\omega^{2} \tau^{2}}+\Gamma_{\mathrm{RG}}\left(\epsilon^{2}, a\right) .
$$

Here we assume an Akhieser mechanism for the anharmonic contribution $\Gamma_{\mathrm{AK}}$, whose strength is proportional to an average Grüneisen constant $\gamma$ and to the specific heat $c_{V}(T)$, while $v_{D}$ is the Debye sound velocity [37-39]. The Rayleigh-Gans term $\Gamma_{\mathrm{RG}}$ is taken from the literature [5], where it is computed in the Born approximation, and is proportional to the variance of the spatial fluctuations of the local sound velocity,
$\epsilon^{2}=\left\langle(\Delta v / v)^{2}\right\rangle . \Gamma_{\mathrm{RG}}$ increases as $\omega^{4}$ up to $\omega \sim v / a$, where $a$ is the correlation length of the fluctuations.

The unknown parameters of Eq. (2), $\gamma, \tau(T), \epsilon^{2}(T)$, $a(T)$, are estimated from a cumulative $\chi^{2}$ fit of the model to the sets of data at the three temperatures, as shown in Fig. 3, where we plot the quantity $2 \pi \Gamma / \Omega^{2}$. The resulting anharmonic contributions are shown as dashed lines in the figure. The low frequency point (full triangle) is measured by means of high-resolution Brillouin light scattering at room temperature and suggests the presence of additional anharmonic processes at low frequencies, likely related to thermally activated relaxations [37]. More details on the light scattering setup can be found in Ref. [52]. The term $\Gamma_{\mathrm{RG}}$ is affected by a mild temperature dependence arising from a weak variation of the correlation length $a$, as shown in the inset of the figure. The analysis indicates that the anharmonic contribution at $100 \mathrm{~K}$ is negligible in the explored frequency range. On the contrary, the decrease of the relaxation time of the Akhieser mechanism at $600 \mathrm{~K}$ $\left(T / T_{g}=0.86\right)$ gives rise to an increases of the low frequency part of the sound attenuation, making it roughly proportional to $\omega^{2}$ in the entire explored frequency range.

The elastic modulus fluctuations responsible for the Rayleigh scattering of the sound waves decay over a correlation length $a$, which is between $50 \%$ and $80 \%$ larger than the silicon-oxygen distance, in agreement with recent findings in silica [3]. Consequently, these fluctuations take place over a distance close to, or slightly larger than, that of an $\mathrm{SiO}_{4}$ tetrahedron, the structural unit responsible for the

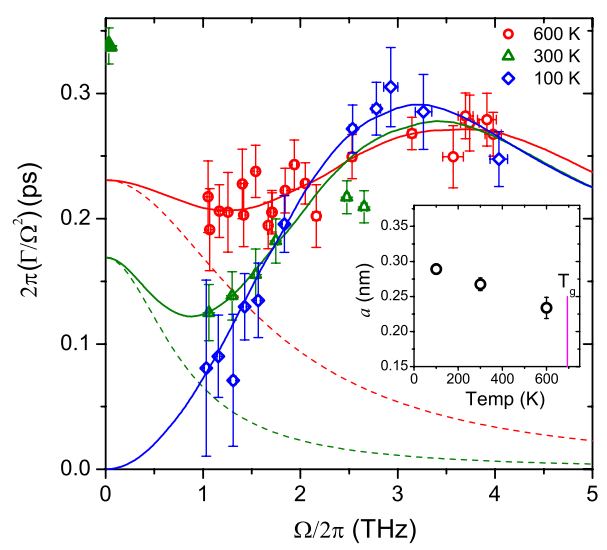

FIG. 3 (color online). $2 \pi\left(\Gamma / \Omega^{2}\right)$ as a function of frequency at the three investigated temperatures (colors and symbols as in Fig. 2). The full triangle (green) is measured by means of Brillouin light scattering with a Fabry-Perot interferometer. The continuous lines are the results of the cumulative $\chi^{2}$ fit to Eq. (2), giving the parameters $\gamma=2.92 \pm 0.22, \tau(300 \mathrm{~K}) \sim 0.2 \mathrm{ps}$, and $\tau(600 \mathrm{~K}) \sim$ $0.1 \mathrm{ps}$. The parameter $\epsilon^{2}$ is temperature independent within experimental uncertainty and is equal to $\epsilon^{2}=0.531 \pm 0.018$. The correlation length $a$ presents a mild temperature dependence, as shown in the inset ( $a$ is the radius in the case of spherical inclusions). The dashed lines are the Akhieser term $\Gamma_{\mathrm{AK}}$ of Eq. (2). The vertical line indicates $T_{g}$. 
medium range order of network glasses. When the frequency exceeds the value $\nu_{c}=v /(2 \pi a)$, the elastic response of the glass becomes very similar to that of the corresponding polycrystal, with excitations related to the acoustic and optic modes of the crystal [3]. It is important to stress that in this regime the Rayleigh-Gans model is only a rough approximation of the sound damping, because the IXS spectrum of the glass is heterogeneously broadened, being composed of more than one excitation, and the parameter $\Gamma$ of the DHO model cannot be regarded as the damping of a single sound wave but only as an estimate of the width of the spectrum.

The observation of a temperature dependence of the sound attenuation also implies a temperature dependence of the DOS. The effect of the anharmonic contribution to the DOS can be estimated using the formula $[15,26]$

$g(\omega)=\frac{2 \omega}{\pi Q_{M}^{3}} \int_{0}^{Q_{M}} q^{2} d q\left\{\operatorname{Im}\left[G_{L}(q, \omega)\right]+2 \operatorname{Im}\left[G_{T}(q, \omega)\right]\right\}$,

where $Q_{M}$ is the maximum value of the wave vector, Im indicates the imaginary part, and $G_{L, T}$ is the phonon propagator for the longitudinal and transverse branches:

$$
G_{L, T}(Q, \omega)=\left[\omega^{2}-Q^{2} \sigma_{L, T}(Q, \omega)\right]^{-1} .
$$

In this expression $\sigma_{L, T}(Q, \omega)$ is a complex self-energy, whose real part is the square of the apparent sound velocity and the imaginary part is related to the sound attenuation through the relation $Q^{2} \operatorname{Im}[\sigma] \sim \omega \Gamma$.

In the Debye approximation $Q_{M}$ is the Debye wave vector and the sound velocity is constant. It is thus natural to assume the self-energy to be $Q$ independent, a choice that allows an analytical solution of the integral in Eq. (3). We also make the hypothesis that $\Gamma_{T}(\omega)=\Gamma_{L}(\omega)$, as suggested by numerical simulations of vitreous silica [53]. The RDOS computed with these assumptions is compared in Fig. 4(a) with that measured by means of inelastic neutron scattering. Details of the determination of the experimental RDOS can be found in Ref. [47]. The model presents a peak in the RDOS which is weaker and displaced to higher frequencies with respect to the measured BP. Moreover, the model predicts that the RDOS goes to the Debye level, dashed horizontal line, at low frequencies, while the measured RDOS remains much higher, presumably because of the presence of additional relaxational processes [54]. The low frequency limit of the computed RDOS depends on the frequency dependence of $\Gamma(\omega)$, since the excess over the Debye level is proportional to $\Gamma / \omega^{2}$ [15]. For this reason, the presence of the Akhieser process implies the computed DOS at low frequencies to be higher than the Debye level. The model predictions for the 300 and $600 \mathrm{~K}$ DOS are shown as dashed and dash-dotted lines and present a variation with respect to the low temperature estimate, which is comparable to that observed in the measured DOS.
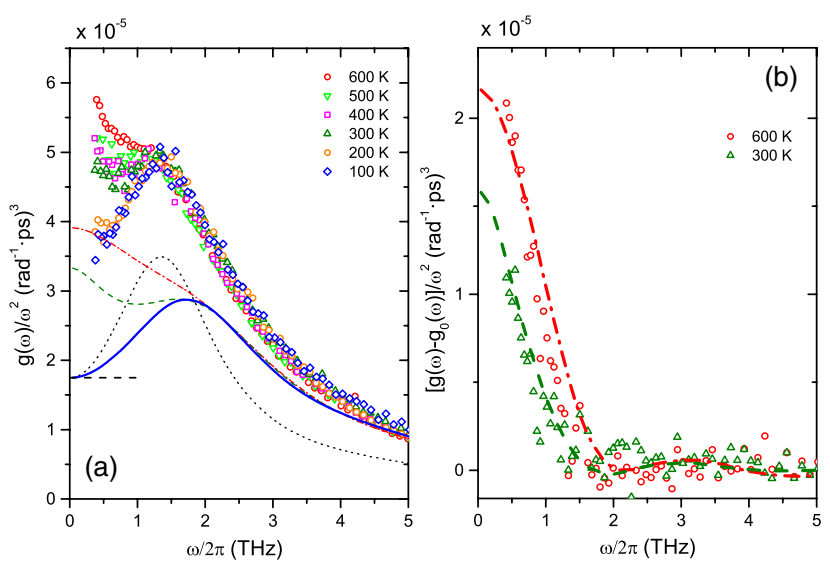

FIG. 4 (color online). (a) RDOS measured by neutron scattering [47] compared to the estimates based on Eq. (3). The dashed horizontal line is the Debye limit, which is temperature independent, as explained in Ref. [47]. Blue continuous line is Eq. (3) at $100 \mathrm{~K}$ under the hypothesis of $\Gamma_{T}(\omega)=\Gamma_{L}(\omega)$. Dashed green and dash-dotted red lines are the same model at 300 and $600 \mathrm{~K}$, respectively. The dotted line is Eq. (3) at $100 \mathrm{~K}$ under the hypothesis of a constant bulk modulus, equivalent to the assumption that $\sigma_{T}(\omega)=(3 / 4) \sigma_{L}(\omega)$. (b) Difference between the RDOS at the indicated temperatures and that at $100 \mathrm{~K}$. The dashed (green) and dash-dotted (red) lines are the ones of (a) minus the continuous blue line.

We can check the generality of the result by varying the hypothesis on the relation between $\Gamma_{T}(\omega)$ and $\Gamma_{L}(\omega)$. The assumption of a frequency-independent bulk modulus, dotted line, is able to well describe the peak position but not its intensity, demonstrating that all models, such as the recent work of Ref. [16], based on Eq. (3) and the hypothesis of a $Q$-independent self-energy, cannot simultaneously reproduce the DOS and the sound attenuation of silicate glasses. The reason for this failure lies in the fact that the model assumes an almost linear dispersion of the acoustic modes up to the Debye frequency, neglecting the contribution coming from a nonlinear dispersion of the acoustic branches, as suggested by the full symbols in Fig. 2(a). However, the model is able to quantitatively describe the effects of temperature on the DOS, as shown in Fig. 4(b), where we plot the difference between the RDOS and the one at $100 \mathrm{~K}, g_{0}(\omega) / \omega^{2}$, at the two indicated temperatures.

In summary, we found evidence of a marked temperature dependence of the sound attenuation of a network glass at terahertz frequencies, a phenomenon that is reported here for the first time. The temperature dependence can be justified quantitatively in terms of the Akhieser mechanism. This attenuation channel is superimposed to a Rayleigh scattering process arising from fluctuations in the local elastic modulus of the material. At low temperatures the Akhieser contribution is negligible and the sound attenuation grows as $\nu^{4}$ in the low frequency range. When the temperature is raised towards $T_{g}$, the combination of the two attenuation mechanisms leads to a sound damping that 
is approximately quadratic in frequency in the entire frequency range. Finally, we have shown the anharmonicity to have a strong effect on the DOS, whose temperature dependence can be evaluated quantitatively in terms of that of the sound attenuation.

The authors acknowledge D. Fioretto for the measurement of the sound attenuation with the Brillouin light scattering technique.

*giacomo.baldi@cnr.it

[1] G. N. Greaves and S. Sen, Adv. Phys. 56, 1 (2007).

[2] H. Wagner, D. Bedorf, S. Küchemann, M. Schwabe, B. Zhang, W. Arnold, and K. Samwer, Nat. Mater. 10, 439 (2011).

[3] G. Baldi, M. Zanatta, E. Gilioli, V. Milman, K. Refson, B. Wehinger, B. Winkler, A. Fontana, and G. Monaco, Phys. Rev. Lett. 110, 185503 (2013).

[4] H. Sato, J. Geophys. Res. 89, 1221 (1984).

[5] J. Kawahara, Wave Motion 48, 290 (2011).

[6] B. Poudel et al., Science 320, 634 (2008).

[7] Z. Wang, J. E. Alaniz, W. Jang, J. E. Garay, and C. Dames, Nano Lett. 11, 2206 (2011).

[8] R. C. Zeller and R. O. Pohl, Phys. Rev. B 4, 2029 (1971).

[9] J. E. Graebner, B. Golding, and L. C. Allen, Phys. Rev. B 34, 5696 (1986).

[10] G. Baldi, V. M. Giordano, G. Monaco, F. Sette, E. Fabiani, A. Fontana, and G. Ruocco, Phys. Rev. B 77, 214309 (2008).

[11] U. Buchenau, Yu. M. Galperin, V. L. Gurevich, and H. R. Schober, Phys. Rev. B 43, 5039 (1991).

[12] D. A. Parshin, H. R. Schober, and V. L. Gurevich, Phys. Rev. B 76, 064206 (2007).

[13] L. E. Silbert, A. J. Liu, and S. R. Nagel, Phys. Rev. Lett. 95, 098301 (2005).

[14] E. Duval, A. Mermet, and L. Saviot, Phys. Rev. B 75, 024201 (2007).

[15] W. Schirmacher, G. Ruocco, and T. Scopigno, Phys. Rev. Lett. 98, 025501 (2007).

[16] S. Köhler, G. Ruocco, and W. Schirmacher, Phys. Rev. B 88, 064203 (2013).

[17] C. Tomaras, B. Schmid, and W. Schirmacher, Phys. Rev. B 81, 104206 (2010).

[18] S. N. Taraskin, Y. L. Loh, G. Natarajan, and S. R. Elliott, Phys. Rev. Lett. 86, 1255 (2001).

[19] A. I. Chumakov et al., Phys. Rev. Lett. 106, 225501 (2011).

[20] A. I. Chumakov et al., Phys. Rev. Lett. 112, 025502 (2014).

[21] B. Rufflé, M. Foret, E. Courtens, R. Vacher, and G. Monaco, Phys. Rev. Lett. 90, 095502 (2003).

[22] B. Rufflé, G. Guimbretière, E. Courtens, R. Vacher, and G. Monaco, Phys. Rev. Lett. 96, 045502 (2006).

[23] G. Monaco and V. M. Giordano, Proc. Natl. Acad. Sci. U.S.A. 106, 3659 (2009).

[24] G. Baldi, V. M. Giordano, G. Monaco, and B. Ruta, Phys. Rev. Lett. 104, 195501 (2010).

[25] B. Ruta, G. Baldi, V. M. Giordano, L. Orsingher, S. Rols, F. Scarponi, and G. Monaco, J. Chem. Phys. 133, 041101 (2010).

[26] G. Baldi, V. M. Giordano, and G. Monaco, Phys. Rev. B 83, 174203 (2011).
[27] C. L. Pekeris, Phys. Rev. 71, 268 (1947).

[28] D. P. Jones, N. Thomas, and W. A. Phillips, Philos. Mag. B 38, 271 (1978).

[29] A. K. Raychaudhuri, Phys. Rev. B 39, 1927 (1989).

[30] S. R. Elliott, Europhys. Lett. 19, 201 (1992).

[31] B. Rufflé, D. A. Parshin, E. Courtens, and R. Vacher, Phys. Rev. Lett. 100, 015501 (2008).

[32] B. Ruta, G. Monaco, V. M. Giordano, F. Scarponi, D. Fioretto, G. Ruocco, K. S. Andrikopoulos, and S. N. Yannopoulos, J. Phys. Chem. B 115, 14052 (2011).

[33] C. Ganter and W. Schirmacher, Phys. Rev. B 82, 094205 (2010)

[34] J. W. S. Rayleigh, The Theory of Sound (MacMillan, London, 1896).

[35] G. Ruocco, F. Sette, R. Di Leonardo, D. Fioretto, M. Krisch, M. Lorenzen, C. Masciovecchio, G. Monaco, F. Pignon, and T. Scopigno, Phys. Rev. Lett. 83, 5583 (1999).

[36] S. Hunklinger and M. v. Schickfus, in Amorphous Solids, Low-Temperature Properties, edited by W. A. Phillips (Springer, Berlin, 1981).

[37] R. Vacher, E. Courtens, and M. Foret, Phys. Rev. B 72, 214205 (2005).

[38] T. O. Woodruff and H. Ehrenreich, Phys. Rev. 123, 1553 (1961).

[39] H. J. Maris, in Physical Acoustics, Principles and Methods, edited by W. P. Mason and R. N. Thurston (Academic, New York, 1971), Vol. VIII.

[40] A. Akhieser, J. Phys. (Moscow) 1, 277 (1939).

[41] H. E. Fischer, F. J. Bermejo, G. J. Cuello, M. T. FernándezDíaz, J. Dawidowski, M. A. González, H. Schober, and M. Jimenez-Ruiz, Phys. Rev. Lett. 82, 1193 (1999).

[42] V. N. Novikov, Phys. Rev. B 58, 8367 (1998).

[43] J. Horbach, W. Kob, K. Binder, and C. A. Angell, Phys. Rev. E 54, R5897 (1996).

[44] J. Horbach, W. Kob, and K. Binder, J. Non-Cryst. Solids 235-237, 320 (1998).

[45] S. N. Taraskin and S. R. Elliott, Phys. Rev. B 59, 8572 (1999).

[46] S. Suzuki, and Y. Abe, J. Non-Cryst. Solids 43, 141 (1981).

[47] G. Baldi, A. Fontana, G. Monaco, L. Orsingher, S. Rols, F. Rossi, and B. Ruta, Phys. Rev. Lett. 102, 195502 (2009).

[48] B. Ruta, G. Baldi, F. Scarponi, D. Fioretto, V. M. Giordano, and G. Monaco, J. Chem. Phys. 137, 214502 (2012).

[49] See Supplemental Material at http://link.aps.org/ supplemental/10.1103/PhysRevLett.112.125502 for details of the model used to analyze the IXS spectra.

[50] L. Orsingher, G. Baldi, A. Fontana, L. E. Bove, T. Unruh, A. Orecchini, C. Petrillo, N. Violini, and F. Sacchetti, Phys. Rev. B 82, 115201 (2010).

[51] H. C. van de Hulst, Light Scattering by Small Particles (Wiley, New York, 1957).

[52] F. Nizzoli and J. R. Sandercock, in Dynamical Properties of Solids, edited by G. Horton and A. A. Maradudin (NorthHolland, Amsterdam, 1990).

[53] S. N. Taraskin and S. R. Elliott, Phys. Rev. B 61, 12031 (2000).

[54] S. Caponi, A. Fontana, F. Rossi, G. Baldi, and E. Fabiani, Phys. Rev. B 76, 092201 (2007). 